\title{
Infant HIV diagnosis and turn-around time for testing in Malawi, 2015
}

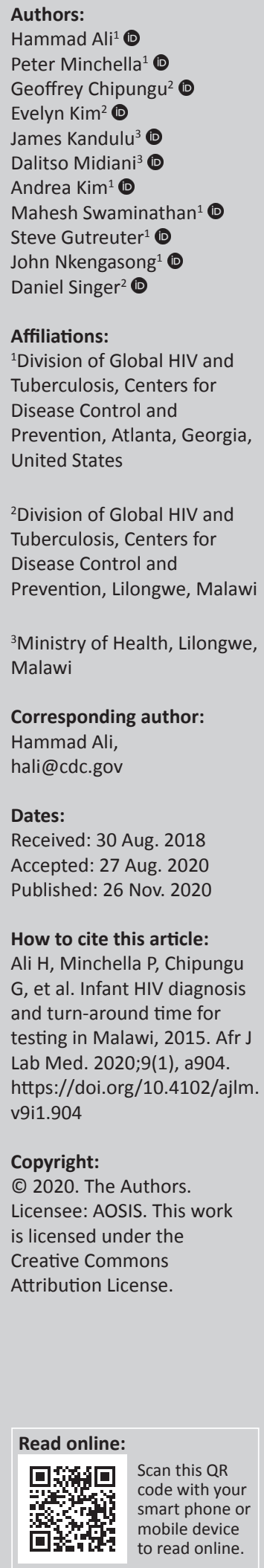

Background: For HIV-exposed infants in Malawi, there are missed opportunities at each step of the testing and treatment cascade.

Objective: This study assessed factors associated with HIV positivity among infants in Malawi and turn-around times for infant HIV testing.

Methods: HIV testing data for infants aged 0-18 months from 2012 to 2015 were extracted from the Malawi HIV laboratory information management system and analysed using logistic regression. Turn-around time was defined as time between collection of samples to results dispatch from the laboratory.

Results: A total of 106997 tests were included in the analyses. A subset of 76006 observations with complete dates were included in the turn-around time analysis. Overall positivity was $4.2 \%$. Factors associated with positivity were increasing age (infants aged 3-6 months: adjusted odds ratio $[\mathrm{aOR}]=2.24$; infants aged $6-9$ months: $\mathrm{aOR}=3.42$; infants aged $>9$ months: $\mathrm{aOR}=$ 4.24), female sex $(\mathrm{aOR}=1.08)$ and whether the mother was alive and not on antiretroviral therapy at time of the infant's test $(\mathrm{aOR}=1.57)$. Provision of HIV prophylaxis to the infant after birth ( $\mathrm{aOR}=0.38$ ) was found to be protective against HIV positivity. The median turn-around time was 24 days (increased from 19 to 34 days between 2012 and 2015).

Conclusion: Infant HIV positivity has decreased in Malawi, whereas turn-around time has increased. Factors associated with positivity include increasing age, female sex, and whether the mother was alive and not on antiretroviral therapy at the time of the infant's test.

Keywords: HIV; Malawi; infant HIV testing; turn-around time.

\section{Introduction}

Malawi has a high prevalence of HIV; in 2016, 9.2\% of persons aged 15-49 years, 920000 people (550 000 women, 60\%) aged 15 years and older, and 110000 children (0-14 years of age) were living with HIV. ${ }^{1}$ Among all people living with HIV, 680000 were on antiretroviral therapy (ART) as of 2016. ${ }^{1}$ Currently, there are 717 sites that offer ART to HIV-positive patients in Malawi and there are nine laboratories nationwide that provide infant HIV virologic testing. ${ }^{2}$ Like many countries in sub-Saharan Africa, Malawi is striving towards achieving the Joint United Nations Programme on HIV / AIDS 90-90-90 targets. These targets include 90\% of people living with HIV diagnosed, $90 \%$ of the people living with HIV with known status accessing treatment, and $90 \%$ of those who are on treatment virally suppressed. ${ }^{3}$ In Malawi, an estimated (antiretroviral adjusted) $76.8 \%$ of $15-64$-year-old people living with HIV know their HIV status, $91.4 \%$ who know their status self-report current use of ART and $91.3 \%$ of those who self-report ART use were virally suppressed. ${ }^{4}$ The second 90 requires 'Test and Start', which is defined as provision of ART for all people living with HIV irrespective of their cluster of differentiation 4 counts. ${ }^{5}$

National guidelines recommend that HIV DNA should be detected using polymerase chain reaction (PCR) in infants who are exposed to HIV. When resources and facilities are available, this should happen at the first opportunity after the infant is older than 6 weeks. ${ }^{6}$ Blood is collected by facility nurses and dried blood spot specimens are created and sent to one of the nine laboratories nationwide that provide infant HIV virologic testing. If the PCR test is negative, a rapid antibody test is done from age 12 months onwards. If the rapid antibody test done at age 12 months returns negative, another rapid antibody test is done at age 24 months, if breastfeeding stopped at least 6 weeks before. If still breastfeeding, then the test is done six weeks after stopping breastfeeding.

In spite of these clear guidelines, HIV diagnosis in HIV-exposed infants has remained a challenge in the country. The Malawi Population-Based HIV Impact Assessment 2015-2016 
showed that around half $(49.4 \%)$ of infants born to HIV-positive women received a virologic HIV test within 2 months of birth, and 29.3\% received a test between 2 and 12 months. ${ }^{4}$ These Malawi PopulationBased HIV Impact Assessment estimates are based on a self-report of the mother and are higher than the estimates from a 2014 cohort study, which showed that only $39 \%-41 \%$ of HIV-exposed infants older than age 24 months had documented evidence of an HIV test result, with $3 \%$ of those tested being positive. ${ }^{2}$ About $82 \%-89 \%$ of those who had a positive result or who were diagnosed with severe HIV disease were initiated on ART. ${ }^{2}$ These data highlight the potential opportunities missed at each step of the testing and treatment cascade for HIV-exposed infants, that is, more infants need to be tested and $10 \%-20 \%$ of those tested positive were not started on ART.

Malawi started universal treatment (i.e., 'Test and Start') for HIV-positive children younger than age 12 months in 2011. In 2014, this was scaled up to cover all children younger than age 5 years, ${ }^{6}$ and in 2016 Malawi implemented 'Test and Start' to cover all people living with HIV nationally. ${ }^{8}$ One component of 'Test and Start' implementation is scaling up of HIV virologic testing among infants born to HIV-positive mothers, and this requires a rapid expansion of laboratory services in Malawi.

There is a paucity of adequate paediatric HIV data generally in Malawi and specifically on factors associated with infant HIV positivity. In addition to difficulties in testing all infants born to HIV-positive mothers, there have been significant challenges in turn-around times for infant HIV test results, thus affecting early diagnosis in Malawi. Turn-around time is a metric used to assess the length of time between steps in the laboratory testing process. Longer turn-around times lead to delays in getting test results, which lead to delayed diagnoses. Any delay in diagnosis invariably leads to delays in the initiation of treatment, leading to poorer health outcomes that can result in higher mortality rates among untreated infants. The objectives of this study were to look at HIV positivity in infants in Malawi over time, and assess factors associated with positivity. In addition, we looked at the turn-around time for infant HIV testing to understand the timeliness of diagnoses.

\section{Methods \\ Ethical considerations}

This study was approved as exempt 45 CFR 46.101(b)(4) by the Centers for Disease Control and Prevention institutional review board (\#6890) and the National Health Science Research Committee, Ministry of Health, Malawi (\#1675).

No extra data were collected for the purpose of these analyses. The Malawi HIV laboratory information management system (LIMS) database does not include patient names but does include unique patient identification numbers, which were scrambled prior to analysis, so that they may be used to clean or de-duplicate the data set but cannot be linked back to a patient.

\section{Study design}

A cross-sectional analysis was conducted on data from the LIMS. The United States President's Emergency Plan for AIDS Relief supported the development of a LIMS in Malawi, which captures basic information on each infant HIV and viral load sample sent for testing. Although originally developed to project reagent needs for laboratories conducting virologic testing and thus keep track of laboratory functions, the database offers important information both on the utilisation of laboratory services as well as on the effectiveness of HIV services. Data included in the LIMS are routinely collected as part of regular provision of HIV services. These data include patient demographics, records of sample transportation and laboratory test outcomes. All data are entered into encrypted databases that are centrally stored and are password protected.

\section{Data analysis}

Data between 2012 and 2015 were extracted from the infant HIV testing database of the Malawi LIMS. Records for infants aged 0 to 18 months at the time of specimen collection were included in the analysis. Tests were excluded from the analysis if the test result was missing. There were two patient identification numbers available in the system that were used. Data were excluded if both patient identification numbers were missing to avoid duplication. Based on the patient identification numbers, if there was more than one record found in the database for a patient identification number, the record with the latest test date was kept and the others dropped before analysis. Infant HIV positivity was defined as detection of HIV DNA using a PCR test. In Malawi, specimens with positive or indeterminate results are re-tested for confirmation. Table 1 shows the variables from the LIMS database that were included in the analysis. Overall turn-around time was defined as the time between collection of the sample at the healthcare facility to the dispatch of the results from the laboratory back to the facility. Data were dropped from the

TABLE 1: Variables extracted from the laboratory information management system to review infant HIV diagnosis and turn-around time for testing in Malawi, 2012-2015.

\begin{tabular}{|c|c|}
\hline Category & Variables included \\
\hline Demographics & Region, age, infant sex \\
\hline $\begin{array}{l}\text { Clinical } \\
\text { information }\end{array}$ & $\begin{array}{l}\text { Infant HIV registration number, HIV care clinic number, multiple- } \\
\text { birth indicator, birth order, infant HIV prophylaxis at birth, infant } \\
\text { ART continued (continuation of ART after completion of } \\
\text { prophylaxis as empiric therapy), infant breast feeding status, } \\
\text { whether mother was alive (on ART or not on ART) or deceased at } \\
\text { the time of the infant test, mother ART status during pregnancy or } \\
\text { labour, reason for PCR testing (e.g., routine vs severe disease) }\end{array}$ \\
\hline $\begin{array}{l}\text { Date of each } \\
\text { step }\end{array}$ & $\begin{array}{l}\text { Date sample drawn at the health facility, date sample received at } \\
\text { lab, date sample tested, date result dispatched back to health } \\
\text { facility }\end{array}$ \\
\hline Test information & Test result \\
\hline
\end{tabular}

ART, antiretroviral therapy; PCR, polymerase chain reaction. 
turn-around time analysis if the date values were implausible (e.g., if the date of the test was before the date of the collection or date of birth). Mean and median turnaround times were calculated between all major steps of the process.

Since the Malawi LIMS includes routinely collected data, a number of variables had missing values. Observations were missing from the records for infant feeding option (60\%), reason for PCR testing (37\%), infant sex (26\%), ART and life status of the mother (alive and on ART, alive but not on ART, deceased; $12 \%)$, multiple-birth indicator $(8 \%)$, region $(1.2 \%)$ and age $(0.2 \%)$. We presumed that observations in all these variables were missing at random and used fully conditional chained equations ${ }^{9}$ to impute 50 completed data sets for analysis.

Bivariate and multivariable logistic regression analyses were undertaken on each of the multiply imputed data sets, and combined using Rubin's rules, ${ }^{10}$ to identify factors associated with infant HIV positivity. The initial multivariable model included all variables approaching significance $(p<0.25)$ in the bivariate model, plus all plausible two-way interactions provisionally detected by changes in odds ratios between the bivariate analysis and a full main-effects model. We eliminated covariates one at a time - beginning with the interactions - based on $p$-values from Wald tests to identify the simplest plausible multivariable model, retaining only covariates and interactions for which $p \leq 0.05$. Our inferences were based upon the adjusted odds ratios (aOR) from the final multivariable model and their 95\% confidence intervals (CI). STATA version 15 (StataCorp LP, College Station, Texas, United States) was used to conduct analyses.

\section{Results}

The 2012-2015 data set included 125713 tests, and 106997 tests $(85.1 \%)$ from unique infants aged 0-18 months which were included in the analysis of associations with HIV positivity. Of those, only 76006 tests had complete date recordings and those were used for the analysis of turnaround times. A total of 23954 tests were conducted in 2012, 27437 in 2013, 29880 in 2014 and 25726 in 2015. Most infants tested were younger than 3 months old (66.6\%); there were almost equal numbers of male (39 628) and female (39 525) infants and most infants were from southern Malawi (69.1\%; Table 2).

\section{HIV positivity}

The overall infant HIV positivity between 2012 and 2015 was $4.2 \%(n=4448)$, which reduced from $5.7 \%$ in 2012 to $3.1 \%$ in 2015. Positivity was $4.3 \%$ in male infants $(n=1701)$ and $4.7 \%$ in female infants $(n=1840)$ and increased by age $(2.3 \%$ in infants aged $<3$ months old, $5.8 \%$ in infants aged 3-6 months old, $9.6 \%$ in infants aged $6-9$ months old, and $12.4 \%$ in infants aged $>9$ months old; Table 2). Positivity was higher if the mother was not on ART during pregnancy or labour (22.6\%) or if the mother's ART status was unknown (4.4\%), compared to women who were on ART during pregnancy or labour (2.5\%). Positivity was highest for infants of deceased mothers (5.6\%), compared to those whose mothers were alive and on ART (2.3\%). Positivity was higher if the infant was not given HIV prophylaxis at birth $(18.1 \%)$ compared to those who were given prophylaxis at birth $(2.5 \%)$.

On multivariable analyses, the odds of testing positive for HIV decreased by $18 \%$ per year (aOR 0.82 , 95\% CI: 0.70 $0.84)$. Other factors significantly associated with testing positive for HIV were increasing age of the infant (3-6 months: aOR 2.24, 95\% CI: 2.07-2.42; 6-9 months: aOR 3.42, 95\% CI: 3.13-3.74; > 9 months: aOR 4.24, 95\% CI: 3.84-4.67), female sex (aOR 1.08, 95\% CI 1.01-1.16), whether the mother was alive but not on ART at the time of the infant HIV test (aOR 1.57, 95\% CI: 1.36-1.82), and if PCR was conducted because the infant was presumed to have severe HIV disease (aOR 2.37, 95\% CI: 1.65-3.39) (Table 2). Immediate postpartum provision of HIV prophylaxis to infants was protective against testing positive for HIV (aOR 0.38, 95\% CI: 0.33-0.44). The effects of ART during pregnancy or labour varied among regions, as manifested by an interaction between region and ART provision during pregnancy or labour. Infants born to mothers who received ART during pregnancy or labour were equally likely to test positive regardless of region. Conversely, the aORs for infants born to mothers who did not receive ART during either pregnancy or labour varied among regions, and infants born in the central region had the lowest aOR (2.80, 95\% CI 2.06-3.81) relative to baseline (infants born in the northern region to mothers who received ART during pregnancy or labour) (Table 3).

\section{Turn-around time}

The overall median turn-around time between HIVrelated sample collection at the healthcare facility to the dispatch of results from the laboratory back to the facility was 24 days (interquartile range: 15-36 days), whereas the mean turn-around time was 29.1 days (standard deviation: 22.5 days) (Table 4 ). The median turn-around time was 19 days in 2012 but increased by 79\% to 34 days in 2015 . Median turn-around time was longest in the northern region (28 days, interquartile range: $20-40$ days), followed by central (27 days, interquartile range: $17-40$ days) and southern region (23 days, interquartile range: $15-35$ days). Median times increased for all steps from 2012 to 2015: median turn-around time between sample drawn at the health facility and being received at the laboratory increased from 11 days in 2012 to 16 days in 2015; the median turn-around time between the sample being received and the sample testing increased from 4 to 6 days; median turn-around time between sample testing and the dispatch of the result from the laboratory back to the facility increased from 0 to 3 days. 
TABLE 2: Population characteristics and factors associated with a positive HIV result in infants in Malawi, 2012-2015. Characteristics

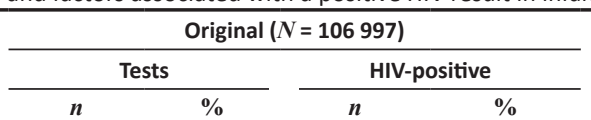

\begin{tabular}{cc} 
& Multip \\
\hline Crude OR* & $95 \% \mathrm{Cl}$ \\
& \\
Ref. & - \\
0.98 & $0.84-1.14$ \\
1.23 & $1.07-1.41$ \\
0.82 & $0.79-0.84$
\end{tabular}

\begin{tabular}{lcccc}
\hline Region & & & & \\
Northern & 6066 & 5.7 & 220 & 3.6 \\
Central & 26634 & 25.2 & 945 & 3.5 \\
Southern & 73000 & 69.1 & 3222 & 4.4 \\
Year & & & &
\end{tabular}

Age

$\begin{array}{llllll}<3 \text { months } & 71127 & 66.6 & 1649 & 2.3\end{array}$

$\begin{array}{lllll}3-6 \text { months } & 21093 & 19.7 & 1215 & 5.8\end{array}$

6-9 months $\quad 8902 \quad 8.3 \quad 856 \quad 9.6$

$\begin{array}{lllll}>9 \text { months } & 5688 & 5.3 & 703 & 12.4\end{array}$

Sex

Male

Female

$39628 \quad 50.1 \quad 1701$

Multiple births

Yes
No
Birth order

Birth order

\begin{tabular}{|c|c|c|c|c|c|c|c|c|}
\hline 0 & 106329 & 99.4 & 4434 & 4.2 & 2.04 & $0.97-4.33$ & - & - \\
\hline 1 & 336 & 0.3 & 7 & 2.1 & Ref. & & - & - \\
\hline 2 & 327 & 0.3 & 7 & 2.1 & 1.03 & $0.36-2.96$ & - & - \\
\hline 3 & 5 & 0.0 & 0 & 0.0 & 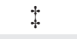 & & - & - \\
\hline \multicolumn{9}{|l|}{ nfant given HIV prophylaxis } \\
\hline No prophylaxis provided & 3561 & 3.3 & 646 & 18.1 & Ref. & & Ref. & - \\
\hline Prophylaxis provided & 39283 & 36.7 & 999 & 2.5 & 0.12 & $0.11-0.13$ & 0.38 & $0.33-0.44$ \\
\hline Status unknown & 64153 & 60.0 & 2803 & 4.4 & 0.21 & $0.19-0.23$ & 0.52 & $0.43-0.63$ \\
\hline \multicolumn{9}{|l|}{ nfant ART continued } \\
\hline No ART provided & 9713 & 9.1 & 772 & 7.9 & Ref. & & - & - \\
\hline ART provided & 30173 & 28.2 & 768 & 2.5 & 0.30 & $0.27-0.33$ & - & - \\
\hline Status unknown & 67111 & 62.7 & 2908 & 4.3 & 0.52 & $0.48-0.57$ & - & - \\
\hline \multicolumn{9}{|l|}{ Feeding options } \\
\hline Ongoing exclusive breastfeeding & 37661 & 88.6 & 1212 & 3.2 & Ref. & & - & - \\
\hline Ongoing complementary feeding & 1332 & 3.1 & 142 & 10.7 & 2.93 & $2.52-3.41$ & - & - \\
\hline Stopped breastfeeding $<6$ weeks & 163 & 0.4 & 15 & 9.2 & 2.16 & $1.35-3.47$ & - & - \\
\hline Stopped breastfeeding $>6$ weeks & 266 & 0.6 & 26 & 9.8 & 2.70 & $1.96-3.74$ & - & - \\
\hline \multicolumn{9}{|l|}{ Mother's ART and life status } \\
\hline Positive - alive on ART & 12363 & 13.1 & 288 & 2.3 & Ref. & - & Ref. & - \\
\hline Positive - alive not on ART & 81983 & 86.8 & 3733 & 4.6 & 1.86 & $1.65-2.10$ & 1.57 & $1.36-1.82$ \\
\hline Dead & 72 & 0.1 & 4 & 5.6 & 2.28 & $0.83-6.28$ & 1.67 & $0.60-4.65$ \\
\hline \multicolumn{9}{|l|}{ ART in pregnancy or labour } \\
\hline ART provided & 38583 & 36.1 & 983 & 2.5 & Ref. & - & Ref. & - \\
\hline No ART provided & 2646 & 2.5 & 599 & 22.6 & 11.19 & $10.02-12.51$ & $\dagger$ & - \\
\hline Status unknown & 65768 & 61.5 & 2866 & 4.4 & 1.74 & $1.62-1.88$ & $\dagger$ & - \\
\hline \multicolumn{9}{|l|}{ Reason for PCR } \\
\hline HIV-exposed infant & 66464 & 99.2 & 2437 & 3.7 & Ref. & - & Ref. & - \\
\hline $\begin{array}{l}\text { HIV-exposed infant with presumed } \\
\text { severe HIV disease }\end{array}$ & 262 & 0.4 & 43 & 16.4 & 3.25 & $2.34-4.52$ & 2.37 & $1.65-3.39$ \\
\hline $\begin{array}{l}\text { Unknown exposure, displaying } \\
\text { symptoms }\end{array}$ & 145 & 0.2 & 11 & 7.6 & 2.09 & $1.21-3.61$ & 0.73 & $0.41-1.30$ \\
\hline
\end{tabular}

ART, antiretroviral therapy; OR, odds ratio; PCR, polymerase chain reaction; Ref., reference category.

*, Bold odds ratio values are significant.

$\dagger$, See interactions.

$\$$, Could not be estimated.

\section{Discussion}

This study used data from Malawi LIMS, a routinely collected HIV data source, and showed that the overall HIV positivity in HIV-exposed infants reduced from $5.7 \%$ in 2012 to $3.1 \%$ in 2015; however, the overall median turn-around time between collection of the sample at the healthcare facility to the dispatch of the results from the laboratory back to the facility increased from 19 days in 2012 to 34 days in 2015. In addition, LIMS data were analysed to identify factors associated with HIV positivity in infants, including infant age and maternal ART uptake. These results highlight the point that routine 
testing data from LIMS can be used not only to inform service delivery but to conduct epidemiological analyses to fill gaps in knowledge about HIV infection.

The positivity results from this study $(3.1 \%$ in 2015$)$ are similar to the results reported by the Ministry of Health (3\% in 2014). ${ }^{2}$ Infant HIV positivity reduced between 2012 and 2015 and prevention of mother-to-child-transmission (PMTCT) initiatives are likely the main reason for this. Specifically, implementation of option B+ (where all pregnant women living with HIV are started on lifelong ART irrespective of their cluster of differentiation 4 count $)^{11}$ - as opposed to option A (in which pregnant women receive ART prophylaxis antenatal and intrapartum, along with a postpartum 'tail' regimen) and option B (in which all women receive ART starting in the antenatal period with continued treatment throughout the breastfeeding duration) - has reduced the mother-to-child-transmission (MTCT) of HIV. The Malawi Population-Based HIV Impact Assessment found that $97.9 \%$ of self-reported HIV-positive women aged 15-49 years who gave birth in the last 12 months before the survey reported having received ART. ${ }^{4}$ It is important to note that Malawi introduced option B+ in 2011, before it became a recommendation by the World Health Organization. ${ }^{12}$ Since then, the coverage of pregnant women who received ART to prevent MTCT increased from $62 \%$ in 2012 to $79 \%$ in 2015 (and further increased to $>95 \%$ in 2018). ${ }^{13}$ Owing to the increase in the number of women who received ART to prevent MTCT, the number of new HIV infections averted have increased, from 8100 in 2012 to 8600 in 2015 (and further increased to 9600 in 2018), and the number of new infections among children aged 0-14 years has decreased from 9300 in 2012 to 6400 in 2015 (and further decreased to 3500 in 2018). ${ }^{13}$ In addition to the increase in PMTCT, the proportion of

TABLE 3: Interaction between antiretroviral therapy in labour or pregnancy and region as factors associated with positive HIV results in infants in Malawi, 20122015.

\begin{tabular}{llcc}
\hline ART in pregnancy or labour & Region & Adjusted odds ratio* & $95 \% \mathrm{Cl}$ \\
\hline ART provided & Northern & Ref. & - \\
& Central & 0.96 & $0.74-1.24$ \\
& Southern & 1.19 & $0.93-1.51$ \\
No ART provided & Northern & 4.07 & $\mathbf{2 . 2 7}-6.09$ \\
& Central & 2.80 & $\mathbf{2 . 0 6 - 3 . 8 1}$ \\
& Southern & 5.35 & $4.05-7.07$ \\
Status unknown & Northern & 1.90 & $1.34-2.70$ \\
& Central & 1.40 & $1.04-1.83$ \\
& Southern & 1.47 & $1.10-1.95$ \\
\hline
\end{tabular}

ART, antiretroviral therapy.

*, Bold values are significant. infants born to HIV-positive women who received an HIV virological test within the first 2 months after birth increased from 5\% in 2012 to $22 \%$ in 2015 (and further increased to $>95 \%$ in 2018). ${ }^{13}$ Thus, the reduction in positivity rates is probably led by the reducing MTCT rates due to option $\mathrm{B}+$. Another possible factor contributing to decreased positivity is the increase in testing in healthy infants due to the expansion of early infant diagnosis testing.

This study identified a number of factors found to be associated with HIV positivity in infants. Increasing age was associated with the risk of testing positive. This could be due to a delay in infant testing, duration of breastfeeding, or lack of access or adherence to care and treatment. Most mothers breastfeed in Malawi and the longer a mother who is not on ART breastfeeds, the greater the risk of transmission of HIV to the infant. ${ }^{14}$ Current Malawi guidelines dictate that HIVexposed infants should be tested between age 6 and 8 weeks. However, this finding shows that at least some of the infants are not getting tested by $6-8$ weeks which may be because mothers are not accessing care and treatment for their HIV. This could be due to non-disclosure issues which can pose a risk for sexual transmission of HIV to the partner, and impact the implementation of PMTCT. ${ }^{15}$ Also, since the latest test result was selected from the LIMS for this study, some results may reflect a repeat or confirmatory test and thus could have contributed to the observed late testing.

Infants born to mothers who received ART during pregnancy or labour experienced indistinguishable risks of HIV infection across all regions. However, infants born to mothers who did not receive ART during either pregnancy or labour were more likely to have been infected, and their odds ratio varied among regions. Infants born in the central region to mothers who did not receive ART had a slightly lower aOR relative to baseline than their counterparts born in the northern and southern regions; however, the confidence intervals overlapped for all three regions. Infants were at higher risk of being tested positive if mothers were alive and not on ART at the time of the test compared to those whose mothers were alive and on ART. This shows the importance of providing ART during pregnancy to women living with $\mathrm{HIV}^{14,16,17,18,19}$ and during labour, ${ }^{18}$ which is well established as a PMTCT measure.

Human immunodeficiency virus-exposed infants not provided with HIV prophylaxis at the time of birth were at an increased risk of being HIV-positive as opposed to those

TABLE 4: Mean and median turn-around time by year for HIV tests in infants in Malawi, 2012-2015.

\begin{tabular}{|c|c|c|c|c|c|c|c|c|}
\hline \multirow{2}{*}{\multicolumn{2}{|c|}{ Median turn-around time between }} & \multirow[t]{2}{*}{ Mean } & \multirow[t]{2}{*}{ Standard deviation } & \multicolumn{5}{|c|}{ Median (interquartile range) } \\
\hline & & & & Overall & 2012 & 2013 & 2014 & 2015 \\
\hline \multicolumn{2}{|c|}{$\begin{array}{l}\text { Overall: Sample drawn at the facility to result } \\
\text { dispatched from the laboratory }\end{array}$} & 29.1 & 22.5 & $24(15-36)$ & $19(12-31)$ & $26(16-37)$ & $24(18-31)$ & $34(18-51)$ \\
\hline Step 1 & $\begin{array}{l}\text { Sample drawn at the facility and received } \\
\text { at the laboratory }\end{array}$ & 15.8 & 15.1 & $12(7-20)$ & $11(7-19)$ & $10(6-18)$ & $14(9-18)$ & $16(8-31)$ \\
\hline Step 2 & Sample received and tested & 9.8 & 10.6 & $6(3-13)$ & $4(2-8)$ & $9(4-21)$ & $5(3-10)$ & $6(3-14)$ \\
\hline Step 3 & $\begin{array}{l}\text { Sample tested and result dispatched from } \\
\text { the laboratory }\end{array}$ & 3.5 & 12.1 & $2(0-3)$ & $0(0-1)$ & $2(1-2)$ & $2(2-4)$ & $3(2-6)$ \\
\hline
\end{tabular}

IQR, interquartile range. 
who were provided with prophylaxis at birth. In addition, HIV-exposed infants with presumed severe HIV disease at The time of the test were at a higher risk of being HIV-positive. Various studies have reported that suboptimal use of ART and inadequate skilled care during the delivery are factors that increase MTCT. ${ }^{14,16}$ Human immunodeficiency virus prophylaxis reduces the concentration of HIV virus in the infants' blood. ${ }^{20,21}$ Thus, HIV prophylaxis should be provided to all exposed infants to reduce perinatal transmission of HIV and those who test positive should be started on ART immediately. Lastly, female infants were at a higher risk of testing HIV-positive compared to male infants. There is some evidence that female infants, compared to male infants, have higher susceptibility to HIV infection before birth. One study reported a significant association between sex and HIV infection, with female infants being at a much higher risk for HIV acquisition than male infants at birth, suggesting in utero acquisition. ${ }^{22}$

Currently, infant HIV virologic testing in Malawi depends on the availability of equipment, laboratory space and trained technicians. ${ }^{23,24}$ Out of the 28 districts in Malawi, only 7 have molecular laboratories that can perform infant HIV virologic testing. Therefore, samples need to be transported to these laboratories for testing from the districts with no molecular laboratories. This can increase the turn-around time for testing, resulting in delays in the return of the result back to the patient and start of treatment if the result is HIV-positive. Multiple factors can prolong turn-around time, including delays with sample transport due to inclement weather, shortage of fuel and holidays. ${ }^{25}$ Laboratory factors can also delay turn-around time, including staff shortages, work overload, stock-out of reagents, issues with equipment and other administrative delays. ${ }^{26}$ The increase in turn-around time documented in this study occurred during a period of rapid expansion of option $\mathrm{B}+$ for PMTCT. The PMTCT programme uses the same mechanisms for transport of samples and ART and the same laboratory facilities for HIV viral load testing. The increased turn-around time likely represents stresses on the system across all levels as scale-up occurred. A sample tracking and online result reporting system - so samples can be tracked during delivery and results can arrive at the facility as soon as tested - can help with improving turn-around time and providing results as soon as possible to clinicians. Another option could be the use of HIV point-of-care testing which can deliver results at the test site. Although point-of-care testing is currently not available on a routine basis in Malawi, a point-of-care early infant diagnosis implementation pilot conducted in Malawi reduced the turn-around time from collection of sample to ART initiation and increased the proportion of patients initiating ART. ${ }^{27}$

The findings in this study are strengthened by the fact that the data set used in this study is the national laboratory data set which includes data from all tests conducted at the national laboratories in the country. This includes tests conducted at the public health facilities and the externally managed facilities and there is a minimal chance of testing happening at a laboratory outside the national laboratories. Additionally, the data are available from 2012 onwards which allows for interpretation of the trends over time. The availability of turn-around time data provides a rare opportunity to monitor the delivery of laboratory services on a national scale. Findings from this study may be applicable throughout sub-Saharan Africa, especially in countries that are scaling up virologic testing.

\section{Limitations}

Like all studies, this study has limitations. Firstly, as the data set only included routinely collected variables, only a limited set of variables could be included in the logistic regression. Some other important variables that could provide insight into other demographic, behavioural and clinical factors associated with HIV positivity in infants could not be included, as they are not collected routinely. Secondly, multiple patient identification numbers were available in the data set. However, none of the identification numbers were completely available for the majority of the patients. Therefore, the data had to be de-duplicated using various patient identification numbers, which, in spite of all efforts, may have led to some duplication in the data set. Thirdly, a number of variables had missing data. This was due to incomplete data recording and changes in the HIV test requisition form over time. We attempted to address this by doing multiple imputations and using the imputed data to conduct the regression analyses. For the turn-around time analysis, nearly $40 \%$ of tests had to be excluded from analysis, if the dates were missing or if the dates were implausible. Better data management and quality control is required to resolve issues of missing data, which will improve the measurement of indicators. Fourthly, the variable 'mother's life and ART status' at the time of the infant's HIV test may not have been correctly entered. It seems that when the mother's life and ART status were missing, it was recorded as mother not on ART. This is important to note, because in the option B+ era in Malawi, it is unlikely that so many women were HIV-positive and not on ART. Lastly, LIMS is not connected to the clinical systems; thus, we were unable to ascertain outcomes of positive tests, that is, what proportion of infants were put on treatment and how quickly.

As LIMS is a routinely collected data source, it can potentially be used for surveillance purposes. However, as described, currently there are a number of data quality and completeness issues that may limit the ability of LIMS to be used as a surveillance system. Attention to detail is required to improve the quality and completeness of LIMS data, and routine data cleaning and validation procedures should be implemented before LIMS can be considered as a surveillance system. In addition, a formal comparison of data generated by LIMS and those generated by other data systems (e.g., electronic medical records) is required to validate the use of LIMS as a surveillance system in Malawi. 


\section{Conclusion}

Although infant HIV positivity has decreased over time in Malawi, the turn-around time has increased over the same period of time. Active engagement from all stakeholders is required to reduce the turn-around time to assist with implementation of 'Test and Start' among infants in the country. The earlier HIV is detected in infants, and treatment started by the clinician, the better the outcomes among these infants. Therefore, it is imperative to test early and provide the results to clinicians as soon as possible so that appropriate care and treatment are provided to infants. Although this study shows that LIMS can provide important information about infant HIV virologic testing, we do not know what proportion of patients actually received these results and what proportion of results were actually acted upon by clinicians. Although LIMS in Malawi was designed to provide information for the laboratories, it can provide other important epidemiological pieces of information. This evidence-based information can guide development and restructuring of policy and programmes in the country.

\section{Acknowledgements}

The authors would like to acknowledge all the implementing partners in Malawi for their support of this study and the mapping exercise. Thanks to Ermias Amene, statistician at the Centers for Disease Control and Prevention, for his assistance.

\section{Competing interests}

No conflicts of interest are declared by authors.

\section{Authors' contributions}

H.A., A.K. and D.S. conceptualised the study; H.A. and S.G. conducted the analysis; H.A. wrote the first draft; P.M., G.C. and A.K. provided technical input; all authors provided input on the initial and final drafts.

\section{Sources of support}

This analysis was supported by the President's Emergency Plan for AIDS Relief through the Centers for Disease Control and Prevention.

\section{Data availability statement}

Data sharing is not applicable to this article as no new data were collected in this study.

\section{Disclaimer}

The findings and conclusions in this report are those of the authors and do not necessarily represent the official position of the funding agencies.

\section{References}

1. Joint United Nations Programme on HIV/AIDS. Malawi HIV and AIDS estimates [homepage on the Internet]. 2017 [cited 2020 Nov 2]. Available from: http:// www.unaids.org/en/regionscountries/countries/malawi/

2. Government of Malawi. Malawi AIDS response progress report 2015. Lilongwe: Government of Malawi; 2015.

3. Joint United Nations Programme on HIV/AIDS. 90-90-90 An ambitious treatment target to help end the AIDS epidemic [homepage on the Internet]. 2014 [cited 2020 Nov 2]. Available from: http://www.unaids.org/en/resources/documents/ 2017/90-90-90

4. Ministry of Health Malawi. Malawi population-based HIV impact assessment (MPHIA) 2015-16: Final report. Lilongwe: Ministry of Health Malawi; 2018.

5. World Health Organization. Guideline on when to start antiretroviral therapy and on pre-exposure prophylaxis for HIV. Geneva: World Health Organization; 2015.

6. Ministry of Health Malawi. Clinical management of HIV in children and adults. Lilongwe: Ministry of Health Malawi; 2014.

7. Ministry of Health Malawi. Clinical management of HIV in children and adults. Lilongwe: Ministry of Health Malawi; 2011.

8. Chinele J. Malawi government says determined on new ART regime. The Times Malawi. 2016 Feb 13 [cited 2020 Nov 2]. Available from: https://times.mw/ malawi-government-says-determined-on-new-art-regime

9. Van Buuren S. Multiple imputation of discrete and continuous data by fully conditional specification. Stat Methods Med Res. 2007;16(3):219-242. https:// doi.org/10.1177/0962280206074463

10. Rubin D. Multiple imputation for nonresponse in surveys. New York, NY: Wiley; 1987.

11. United Nations Children's Fund. Options B and B+: Key considerations for countries to implement an equity-focused approach. New York, NY: UNICEF; 2012.

12. Kieffer MP, Mattingly $M$, Giphart $A$, et al. Lessons learned from early implementation of option B+: The Elizabeth Glaser Pediatric AIDS Foundation experience in 11 African countries. J Acqui Immune Defic Syndr. 2014;67(Supp 4):S188. https://doi.org/10.1097/QAl.0000000000000372

13. Joint United Nations Programme on HIV/AIDS [homepage on the Internet]. AIDSinfo. 2019 [cited 2020 Nov 2]. Available from: http://aidsinfo.unaids.org/

14. Berhan Z, Abebe F, Gedefaw M, Tesfa M, Assefa M, Tafere Y. Risk of HIV and associated factors among infants born to HIV positive women in Amhara region, Ethiopia: A facility based retrospective study. BMC Res Notes. 2014;7(1):876. https://doi.org/10.1186/1756-0500-7-876

15. Hatcher AM, Woollett N, Pallitto CC, et al. Bidirectional links between HIV and intimate partner violence in pregnancy: Implications for prevention of mother-tochild transmission. J Int AIDS Soc. 2014;17(1):19233. https://doi.org/10.7448/ IAS.17.1.19233

16. Anígilájé $\mathrm{E}$, Dabit $\mathrm{O}, \mathrm{Ageda} \mathrm{B}, \mathrm{Hwande} \mathrm{S}$, Bitto T. The prevalence and predictors of HIV infection among children of mothers who missed prevention of mother to child transmission of HIV interventions in Makurdi, Nigeria. J AIDS Clin Res. 2013;4(11):249. https://doi.org/10.4172/2155-6113.1000249

17. Magder LS, Mofenson L, Paul ME, et al. Risk factors for in utero and intrapartum transmission of HIV. JAIDS. 2005;38(1):87-95. https://doi.org/10.1097/00126334200501010-00016

18. De Lemos LM, Lippi J, Rutherford GW, et al. Maternal risk factors for HIV infection in infants in northeastern Brazil. Int J Inf Dis. 2013;17(10):e913-e918. https://doi. org/10.1016/j.ijid.2013.04.015

19. Okoko NA, Owuor KO, Kulzer JL, et al. Factors associated with mother to child transmission of HIV despite overall low transmission rates in HIV-exposed infants in rural Kenya. Int J STD AIDS. 2017;28(12): 1215-1223. https://doi. org/10.1177/0956462417693735

20. De Cock KM, Fowler MG, Mercier E, et al. Prevention of mother-to-child HIV transmission in resource-poor countries: Translating research into policy and practice. JAMA. 2000;283(9):1175-1182. https://doi.org/10.1001/jama.283.9.1175

21. Volmink J, Siegfried N, van der Merwe L, Brocklehurst P. Antiretrovirals for reducing the risk of mother-to-child transmission of HIV infection. Cochrane Database Syst Rev. 2007;1:CD003510. https://doi.org/10.1002/14651858.CD003510.pub2

22. Taha TE, Nour S, Kumwenda NI, et al. Gender differences in perinatal HIV acquisition among African infants. Pediatrics. 2005;115(2):e167-e172. https:// doi.org/10.1542/peds.2004-1590

23. Fiscus SA, Cheng B, Crowe SM, et al. HIV-1 viral load assays for resource-limited settings. PLoS Med. 2006;3(10):e417. https://doi.org/10.1371/journal.pmed. 0030417

24. Roberts T, Bygrave H, Fajardo E, Ford N. Challenges and opportunities for the implementation of virological testing in resource-limited settings. J Int AIDS Soc. 2012;15(2):17324. https://doi.org/10.7448/IAS.15.2.17324

25. Minchella PA, Chipungu G, Kim AA, et al. Specimen origin, type and testing laboratory are linked to longer turnaround times for HIV viral load testing in Malaw PLoS One. 2017;12(2):e0173009. https://doi.org/10.1371/journal.pone.0173009

26. Thiha S, Shewade HD, Philip S, et al. Factors associated with long turnaround time for early infant diagnosis of HIV in Myanmar. Glob Health Action. 2017;10(1):1395657. https://doi.org/10.1080/16549716.2017.1395657

27. Mwenda R, Vojnov L, Saka E, et al. Significantly improved antiretroviral therapy initiation rates after the implementation of point of care early infant diagnosis. African Society for Laboratory Medicine Conference Cape Town, South Africa. 3-8 December 2016 